\title{
Comparative Study of Three Different Path Tracking Controls in Mobile Robots
}

\author{
Abel García-B., Cristian \\ G. Pérez-T., Eduardo S. \\ Espinoza-Q., and \\ Francisco R. Trejo-M. \\ Department of Mechatronics, \\ Polytechnic University of \\ Pachuca, Zempoala, Hidalgo, \\ Mexico
}

\author{
Luis E. Ramos-V. and \\ Hugo Romero-T., \\ Research Center for \\ Information Technologies and \\ Systems, Autonomous \\ University of Hidalgo State \\ (UAEH), Hidalgo, Mexico
}

\author{
Jesús M. Muñoz-P. \\ Department of Electronics, \\ Meritorious Autonomous \\ University of Puebla (BUAP), \\ Puebla, Mexico
}

\begin{abstract}
This paper presents a comparative study of three different path tracking control laws for the formation of a group of nonholonomic mobile robots. By introducing a unified error of the formation and trajectory tracking using; the dynamic feedback linearization control [1], dynamic-static feedback linearization control [2] and nonlinear time-invariant control [3] are compared. The simulations results show that the dynamic-static feedback linearization technique presents a stable tracking with smoother behaviour and avoiding discontinuities for tracking trajectory of the robot leader. Finally, this method was implemented experimentally in three different paths formatting a simple triangle with three mobile robots in a leader-follower type motion. Moreover, the analysis in this paper reveals some important issues raising that the following control on this system can be extended to underactuated AUVs in future work.
\end{abstract}

\section{Keywords}

Multiple robot system; formation path tracking; nonlinear control law.

\section{INTRODUCTION}

Over the last decades, a lot of attentions have been paid to research on the cooperative control and formation control of multiple robots, especially on mobile robots. The collective nature of multi robot systems attracts many vibrant real world applications. It includes exploration, surveillance, cooperative large objects, cooperative attack and rendezvous and formation control [1-8]. With increasing popularity and interest in the vision of multirobots systems, there are many topics with huge potential to make these mechatronics systems possible in real life applications [9]. There is currently considerable interest in the problem of coordinated motion control to carry out a specific task of cooperation, i.e. to move a heavy object. Different control strategies have been proposed [1-3], which include the behaviour based, virtual structure, leader following, and graph theoretical approaches. While most existing results use linear vehicle dynamics to simplify control design, we study formation control of nonhomolomic mobile robots. Formation control means the problem of controlling the relative position and orientation of the mobile robots in a group according to some desired patter for executing a given task [10-11]. Trajectory tracking is required to enable the robot a time parameterized reference path. Path following drives the mobile robot to converge to a follow a desired spatial path, without strict temporal specifications. Also, different approaches have been proposed and implemented to tackle the robot formation control problem [6-9]. Typically, smoother convergence to a path is achieved when path following strategies are used instead of trajectory tracking control laws, and the control signals are less likely pushed to saturation. The most common techniques used are the following: dynamic feedback linearization control [1], a dynamic-static feedback linearization control [2] and nonlinear time-invariant control [3]. The dynamic and dynamic-static feedback linearization techniques are used to derive the formation control laws for follower robots that are used for leader-following formation. The nonlinear timeinvariant feedback control is another technique to carry out the formation of multi robots systems.

In this paper, we present the comparison of three different path-tracking controls for the formation of mobile robots. Nonlinear control laws to tracking paths on a Cartesian plane and maintaining the desired formations are simulated and implemented. The simulation path of the mobile robot is carried out by using the Matlab toolbox animation. The technique of input-output linearization by dynamic-static feedback control for trajectory tracking and formation control was implemented experimentally in this paper. The remainder of the paper is structured as follow. In Section II the kinematic models of nonholomic mobile robots are presented, in Section III the control laws compared in this paper are presented. Simulation and experimental results of the formation robots and path tracking are presented in Section IV. Finally, in Section V, we present the conclusions of this research.

\section{KINEMATIC MODELS}

A collaborative robot system can be described by its sate which is a composition of the individual robots, as usually it is studied. The state of each robot varies as a function of its own state as well as the information sent by the order robots. There are various approaches used for formation control of collaborative robots, and these can be roughly divided into three categories: leader-follower formation, behavior based and virtual structure formation [12-13]. In this paper, the leader-follower formation is used, in which the follower robots maintain a desired distance and a desired bearing angle relative to the leader robot. In order to simplify the kinematic model and the control law, we consider the nonholonomic constrains which arise from constraining each pair of wheels roll without slipping [14] and we assume that the mobile 
robots under study in this paper are made up of rigid frame equipped with non-deformable wheels and they are moving on a horizontal plane. Figure 1 shows the schematic diagram of wheeled mobile robot with two driven wheels (in the rear part) and a passive castorwheel (in the front).

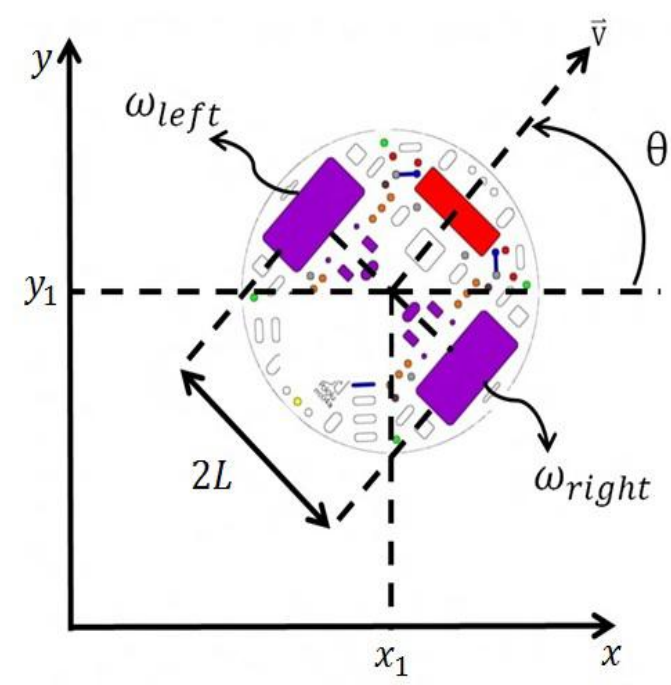

Figure 1: Schematic diagram of the mobile robot

The state-space model of the considered kinematic vehicle with the associated nonholonomic constraints (rolling with no slipping) is given by,

$$
\begin{gathered}
\dot{x}=\frac{r\left(\omega_{\text {left }}+\omega_{\text {right }}\right) \cos (\theta)}{2} \\
\dot{y}=\frac{r\left(\omega_{\text {left }}+\omega_{\text {right }}\right) \sin (\theta)}{2} \\
\dot{\theta}=\frac{r\left(\omega_{\text {left }}-\omega_{\text {right }}\right)}{2 L}
\end{gathered}
$$

where $r$ is the radius of the tire, $L$ is the distance between the tire and the centre of the robot, $\omega_{\text {left }}$ and $\omega_{\text {right }}$ are the angular velocity of the left and the right wheel, respectively. The robot's position is completely specified by three variables $x, y$ and $\theta$, see Figure 1. Also, $x_{1}$ and $y_{1}$ represent the position along the axis $\mathrm{X}$ and $\mathrm{Y}$, respectively, and $\theta$ represents the orientation of the longitudinal axis of the robot with respect to the axis X. In matrix form, the velocities can be expressed as

$$
\left[\begin{array}{l}
\dot{x} \\
\dot{y} \\
\dot{\theta}
\end{array}\right]=\left[\begin{array}{cc}
\cos (\theta) & 0 \\
\sin (\theta) & 0 \\
0 & 1
\end{array}\right]\left[\begin{array}{l}
v_{1} \\
\omega_{1}
\end{array}\right]
$$

where $v_{1}$ and $\omega_{1}$ are the linear and angular velocity, respectively, and $\theta$ is the robot orientation, which represent the unicycle-type mobile robot model, the relations between $v_{1}, \omega_{1}$ and $\omega_{\text {left }}, \omega_{\text {right }}$ are given by,

$$
\left[\begin{array}{c}
v_{1} \\
\omega_{1}
\end{array}\right]=\left[\begin{array}{cc}
r / 2 & r / 2 \\
r / 2 L & -r / 2 L
\end{array}\right]\left[\begin{array}{c}
\omega_{\text {left }} \\
\omega_{\text {right }}
\end{array}\right]
$$

In this paper, we consider a networked team of three nonholonomic unicycle-type mobile vehicles, labelled 1 trough 3, tracking a set of paths, while attaining a desired formation. First, we consider a two 3-wheel robot system, the coordinate positions of these two robots are shown in Figure2. It is a system of two mobile robots separated for a distance $l_{12}$ between the centre of the first robot and the balance ball of the second robot, $d$ denotes the distance between the balance ball and the axis of the wheels of each robot. The kinematics equations, obtained from Figure 2, show that the system states variables are given by $l_{12}, \psi_{12}$, and $\theta_{2}$. Then, the kinematic equations for a single robot are given by Eq. (4), while and kinematic model for two robots is given by

$$
\begin{gathered}
{\left[\begin{array}{c}
\dot{l}_{12} \\
\dot{\psi}_{12} \\
\dot{\theta}_{2}
\end{array}\right]=\left[\begin{array}{cc}
\cos \left(\gamma_{1}\right)-\cos \left(\psi_{12}\right) & 0 \\
1 / l_{12} \sin \left(\psi_{12}\right) & 1 \\
0 & 0
\end{array}\right]\left[\begin{array}{l}
v_{1} \\
\omega_{1}
\end{array}\right]} \\
+\left[\begin{array}{cc}
0 & d \cos \left(\gamma_{1}\right) \\
-1 / l \sin \left(\gamma_{1}\right) & 1 / l_{12} d \cos \left(\gamma_{1}\right) \\
0 & 1
\end{array}\right]\left[\begin{array}{l}
v_{1} \\
\omega_{1}
\end{array}\right]
\end{gathered}
$$

where $\gamma_{1}=\theta_{1}+\psi_{12}-\theta_{2}$. One can see the states variables to control according to the kinematic Eq. (6), and then it is possible to get the proper control scheme.

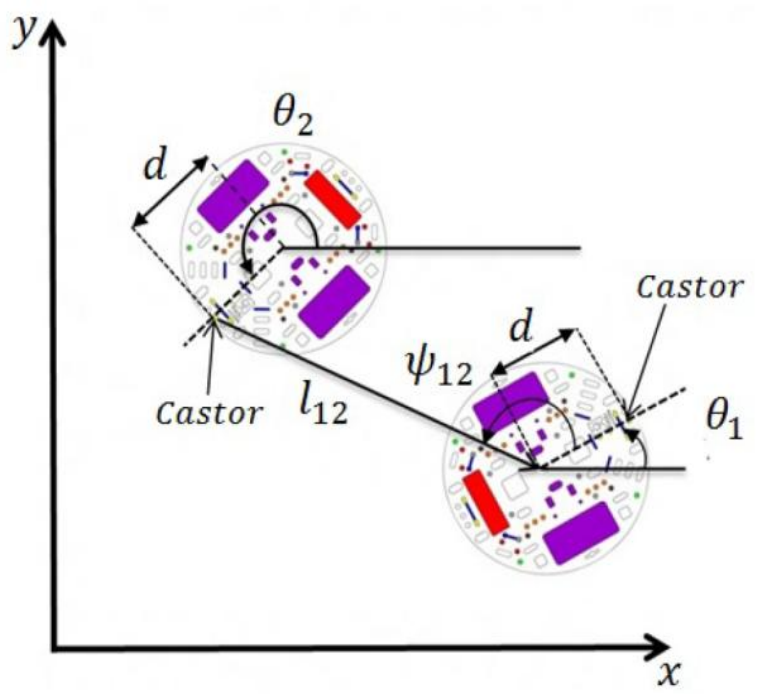

Figure 2: Schematic diagram for two mobile robots

Below, we presented an approach for tackling the rigid formation control problem by employing the flatness property. An effective control strategy allows us to execute formation maneuvers, departing from the formation, splitting the formation, and merging into formation.

\section{MOBILE ROBOT CONTROL}

In the path tracking control of mobile robots, a nonholomomic unicycle-type of wheeled vehicle follows a predefined spatial path, $\mathrm{X}_{\mathrm{d}}=\left[x(\mathrm{t})_{\mathrm{d}} ; y(\mathrm{t})_{\mathrm{d}} ; \theta(\mathrm{t})_{\mathrm{d}}\right]$. In this work, we move a mobile robot along three desire paths described by the following equations

$$
\begin{gathered}
y(t)_{d 1}=x(t)_{d} \\
y(t)_{d 2}=x^{3}(t)_{d} \\
y(t)_{d 3}=\sqrt{r^{2}-\left(x(t)_{d}-a\right)^{2}}+b
\end{gathered}
$$

where Eqs. (7) describe a straight line, a hyperbola, and a semicircle path, respectively. In Eq. (7c), $a$ and $b$ represent the origin point of the semicircle. Deriving Eqs. (7) with respect to time and substituting in (4), we found that the orientation angle is given by the following equations

$$
\begin{gathered}
\theta(t)_{d 1}=\arctan (C) \\
\theta(t)_{d 2}=\arctan \left(3 x^{2}(t)_{d}\right) \\
\theta(t)_{d 3}=\arctan \left(\sqrt{r^{2}-\left(x(t)_{d}-a\right)^{2}}\left(x(t)_{d}-a\right)\right)
\end{gathered}
$$


where $C$ is the relation between $x_{\mathrm{d}}$ and $y_{\mathrm{d}}$. The next step is to define $x(t)_{\mathrm{d}}$, which is given by

$$
x(t)_{d}=a \sin \left(\frac{2 \pi}{P} t\right)
$$

Using Eq. (9), the mobile robot goes from the origin at $x=0$, $y=0$ to the point $x=a, y=a^{3} \mathrm{~m}$, then it returns to the origin and moves to the point $x=-a, y=-a^{3} \mathrm{~m}$, and finally it backs again to the origin point, in the case of the second path and it will be ending in $P$ seconds.

Dynamic Feedback Control. In this method, it is developed a controller that linearizes the input - output system response. Considering $u_{1}$ and $u_{2}$ as control variables, $x$ and $y$ as output variables [1], it is possible to represent the system in the standard form $\dot{q}=A(\theta) u$

$$
\left[\begin{array}{c}
\ddot{x} \\
\ddot{y}
\end{array}\right]=\left[\begin{array}{ll}
\cos (\theta) & 0 \\
\sin (\theta) & 0
\end{array}\right]\left[\begin{array}{l}
u_{1} \\
u_{2}
\end{array}\right]=A(\theta)\left[\begin{array}{l}
u_{1} \\
u_{2}
\end{array}\right]
$$

One can see that the matrix $A(\theta)$ is singular for all values of $x, y$, and $\theta$, therefore it would not be possible to linearize the system. For this reason, we need a new state variable $\varsigma$ and also a control variable, $\tilde{u}_{1}$, where the change of variables are $\dot{\zeta}=\tilde{u}_{1}$ and $u_{1}=\varsigma$. The new control variables $\tilde{u}_{1}$ and $u_{2}$ are related with the output variables as follow

$$
\left[\begin{array}{l}
\ddot{x} \\
\ddot{y}
\end{array}\right]=\left[\begin{array}{ll}
\cos (\theta) & \varsigma \sin (\theta) \\
\sin (\theta) & \varsigma \cos (\theta)
\end{array}\right]\left[\begin{array}{l}
\tilde{u}_{1} \\
u_{2}
\end{array}\right]=A(\theta, \varsigma)\left[\begin{array}{l}
\tilde{u}_{1} \\
u_{2}
\end{array}\right]
$$

If $\varsigma \neq 0$, then it is possible to rewrite the feedback system as follow

$$
\left[\begin{array}{l}
\tilde{u}_{1} \\
u_{2}
\end{array}\right]=A^{-1}(\theta, \varsigma)\left[\begin{array}{l}
v_{1} \\
v_{2}
\end{array}\right]
$$

the new control variables are $v_{1}$ and $v_{2}$ are given by

$$
\left[\begin{array}{l}
v_{1} \\
v_{2}
\end{array}\right]=\left[\begin{array}{l}
\ddot{x}_{d}-k_{x 1} \dot{e}_{x}-k_{x 0} e_{x} \\
\ddot{y}_{d}-k_{y 1} \dot{e}_{y}-k_{x 0} e_{x}
\end{array}\right]
$$

where the constants $k_{\mathrm{xi}}$ and $k_{y i}$ represent the control gains, which make the error tends to converge to zero, and the error functions are defined by $e_{\mathrm{x}}=x_{\mathrm{d}}-x$ and $e_{\mathrm{y}}=y_{\mathrm{d}}-y$.

Dynamic-static Feedback Linearization Control. The control law in Eq. (12), clearly, it is not defined when $\varsigma=0$. For this reason, we need to propose an static feedback control law that linearizes the input - output system [2], but with the output variables $x$ and $\theta$. From Eq. (4) we can see that the control variables are related with the output variables as follow

$$
\left[\begin{array}{l}
\dot{x} \\
\dot{\theta}
\end{array}\right]=\left[\begin{array}{cc}
\cos (\theta) & 0 \\
0 & 1
\end{array}\right]\left[\begin{array}{l}
u_{1} \\
u_{2}
\end{array}\right]=\tilde{A}(\theta)\left[\begin{array}{l}
u_{1} \\
u_{2}
\end{array}\right]
$$

where the control variables $v_{1}$ and $v_{2}$ were proposed as

$$
\left[\begin{array}{l}
u_{1} \\
u_{2}
\end{array}\right]=\tilde{A}^{-1}(\theta, \varsigma)\left[\begin{array}{l}
v_{1} \\
v_{2}
\end{array}\right]
$$

where the control variables $v_{1}$ and $v_{2}$ are defined as

$$
\left[\begin{array}{l}
v_{1} \\
v_{2}
\end{array}\right]=\left[\begin{array}{l}
\dot{x}_{d}-k_{x} e_{x} \\
\dot{\theta}_{d}-k_{\theta} e_{x}
\end{array}\right]
$$

where the constants $k_{\mathrm{x}}$ and $k_{\theta}$ are the control gains, which make the error to converge to zero and the error functions are defined by $e_{\mathrm{x}}=x-x_{\mathrm{d}}$ and $e_{\theta}=\theta-\theta_{d}$.

The control law, in Eq. (15), is not defined for $\theta=k \pi / 2, k=$ $\pm 1 ; \pm 2 ; \ldots$ as it grows out of control, and as mentioned before the control law, Eq. (12) is not defined when $\varsigma=0$, i.e. when the robot is at rest or when the path indicates to the robot, that must it stops for a moment. For these reasons, we can say that the control laws are not defined globally, and switching control given by Eq. (12), and the Eq. (15) is employed to avoid discontinuities.

Nonlinear Time-Invariant Control. The tracking controller given by [3] was obtained from a Lyapunov based design as

$$
\begin{aligned}
& v=v_{d} \cos \left(\theta_{d}-\theta\right)+k_{1}\left[\left(x_{d}-x\right) \cos (\theta)+\left(y_{d}-\right.\right. \\
& \text { y) } \sin (\theta)] \\
& \omega=\omega_{d}+k_{2} v_{d} \frac{\sin \left(\theta_{d}-\theta\right)}{\theta_{d}-\theta}\left[\left(y_{d}-y\right) \cos (\theta)-\left(x_{d}-\right.\right. \\
& \text { x) } \sin (\theta)]+k_{3}\left(\theta_{d}-\theta\right)
\end{aligned}
$$

where $v_{\mathrm{d}}$ and $\omega_{\mathrm{d}}$ are defined by the following equations

$$
\begin{gathered}
v_{d}= \pm \sqrt{\dot{x}_{d}^{2}+\dot{y}_{d}^{2}} \\
\omega_{d}=\frac{\ddot{y}_{d} \dot{x}_{d}-\ddot{x}_{d} \dot{y}_{d}}{\dot{x}_{d}^{2}+\dot{y}_{d}^{2}}
\end{gathered}
$$

A common technique to calculate the control gains is by using

$$
\begin{gathered}
k_{1}=k_{3}=2 \varsigma \sqrt{\omega_{d}^{2}+b v_{d}^{2}} \\
k_{2}=b
\end{gathered}
$$

with damping coefficient $\varsigma \in(0,1)$ and $b>0$.

\section{RESULTS}

We carried out the comparative study of three control laws, described in the last section, and maintained a triangular formation. In this case, we chose the leader-follower technique, with a leader and two robots followers. We use a controller that linearizes the response input-output system, using a control law that gives exponentially convergent to the variables $l_{12}$ and $\psi_{12}$, between robots 1 and 2 , and the variables $l_{13}$ and $\psi_{13}$, between robots 1 and 3 , with control law defined by Eq. (24)

$$
\begin{gathered}
\omega_{i}=\cos \left(\lambda_{d l i}\right)\left[\alpha_{i} l_{1 i}\left(\psi_{d 1 i}-\psi_{1 i}\right)-v_{1} \sin \left(\psi_{1 i}\right)+l_{1 i} \omega_{1}+\right. \\
\left.\rho_{l i} \sin \left(\lambda_{l i}\right)\right] \\
v_{i}=\rho_{l i}-d \omega_{i} \tan \left(\lambda_{l i}\right)
\end{gathered}
$$

where

$$
\rho_{l i}=\frac{\alpha_{1}\left(l_{d 1 i}-l_{1 i}\right)+v_{1} \cos \left(\psi_{l i}\right)}{\cos \left(\lambda_{l i}\right)}
$$

where $\gamma_{1 \mathrm{i}}=\theta_{1}+\psi_{1 \mathrm{i}}-\theta_{\mathrm{i}}, v_{\mathrm{i}}$ and $\omega_{i}$ are the linear and angular velocities for each follower robot for $i=2,3$. This leads the error dynamics in the variables $l-\psi$ variables in the form

$$
\begin{aligned}
& l_{1 i}=\alpha_{i}\left(l_{d 1 i}-l_{1 i}\right) \\
& \dot{\psi}_{l i}=\alpha_{i}\left(\psi_{d l i}-\psi_{l i}\right)
\end{aligned}
$$

The simulation results of path tracking control with a comparison of three different control laws and different trajectories with a triangular formation using three mobile robots are obtained. The control scheme of trajectory following and the constant formation was simulated using Matlab Simulink, with a simulation time of 60 seconds. First, we first consider the reference trajectory is a circle path, it is showed in Figure 3, which starts at $x=1 \mathrm{~m}, y=1 \mathrm{~m}$ with a radius of $0.5 \mathrm{~m}$. The robot is initially at $x=0.3 \mathrm{~m}, y=0.2 \mathrm{~m}, \theta$ $=0 \mathrm{rad}$. Figure 3 shows that, although an stable tracking is obtained using the dynamic feedback and dynamic-static feedback techniques, when the robot is at rest occurs the discontinuity $\varsigma=0$, the dynamic feedback control does not 
avoid this discontinuity. In the case of the nonlinear timeinvariant controller controller does not appear the discontinuity $\varsigma=0$, but the controller has a non smoother behavior, therefore the dynamic-static feedback control works efficiently because it has stable tracking, an smoother behavior and avoid the discontinuity $\varsigma=0$.

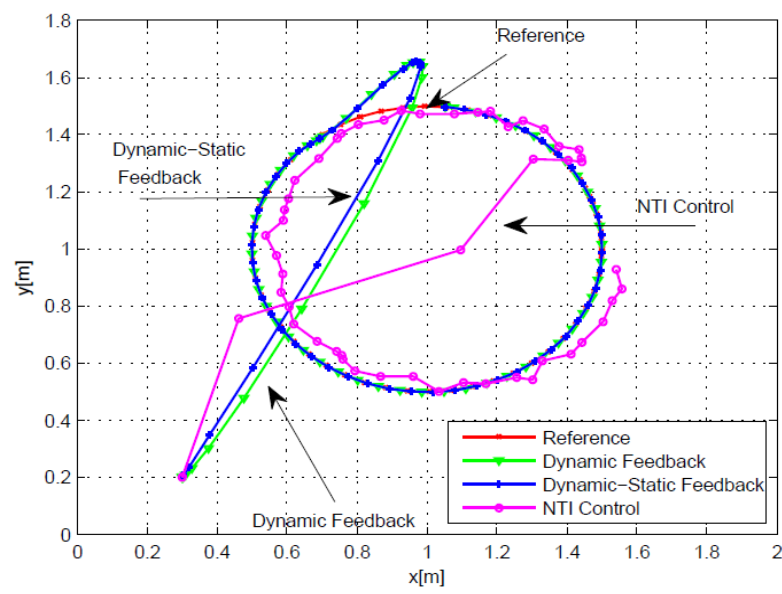

Figure 3: Simulation results of path tracking controllers

In order to obtain an efficiently path, we consider that the leader robot plans a trajectory given by the dynamic-static feedback controller and two follower robots which overall the leader realize a triangular formation. Figure 4 shows the leader robot performing a straight line path which goes from the origin at $x=0 \mathrm{~m}, y=0 \mathrm{~m}$ to the point $x=0.5 \mathrm{~m}, y=0 \mathrm{~m}$, then it returns to the origin and moves to the point $x=0.5 \mathrm{~m}, y$ $=0 \mathrm{~m}$, and finally it backs again to the origin, is the same for the two follower robots. In order to obtain a triangular formation, we chose $\psi_{12}=60^{\circ}, l_{12}=2 d$ for the robot follower 1 and $\psi_{13}=-60^{\circ}, l_{13}=2 d$ for the robot follower 2 , where $\mathrm{d}=$ $0.06 \mathrm{~m}$ is the distance between the castor and the center of the axes of the wheels. One can see a smoother behavior of the two follower robots, see Figure 4. Also, it is observed peaks in both paths, because the follower robots change of orientation maintaining the framework orientation in entire path.

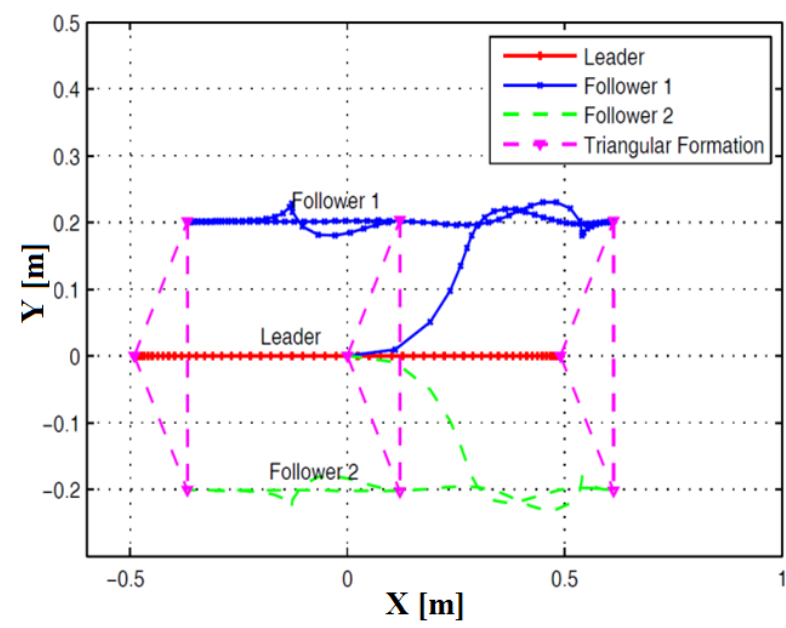

Figure 4: Straight line trajectory of the leader robot with two follower robots which overall realize a triangular formation

The second path of the leader robot is a hyperbolic trajectory showed in the Figure 5, which goes from $x=0 \mathrm{~m}, y=0 \mathrm{~m}$ to the point $x=0.5 \mathrm{~m}, y=0.12 \mathrm{~m}$, then it returns to the origin and moves to the point $x=-0.5 \mathrm{~m}, \mathrm{y}=-0.12 \mathrm{~m}$, and finally it comes back again to the origin. Figure 5 shows an efficiently path of the leader robot with two follower robots which are maintaining the triangular formation along entire path, each follower robot changes of orientation when the leader robot comes back to the origin.

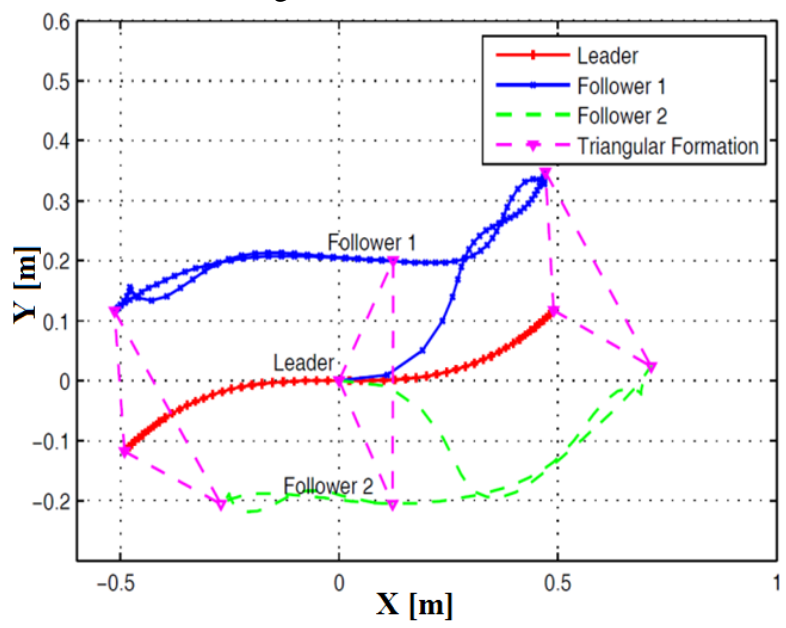

Figure 5: Hyperbolic trajectory of the leader robot with two follower robots which overall realize a triangular formation

The last trajectory to follow by the leader robot is the semicircle showed in the Figure 6, which goes from $x=0 \mathrm{~m}, y$ $=0 \mathrm{~m}$ to the point $x=0.5 \mathrm{~m}, y=0.4 \mathrm{~m}$, then it returns to the origin and moves to the point $x=-0.5 \mathrm{~m}, y=-0.4 \mathrm{~m}$ and finally, it comes back again to the origin. Figure 6 shows an efficiently semicircular path tracked by the leader robot with two follower robots, which are maintaining the triangular formation along entire path, proving that it can realize any efficiently trajectory at time the follower robot maintaining the desire formation.

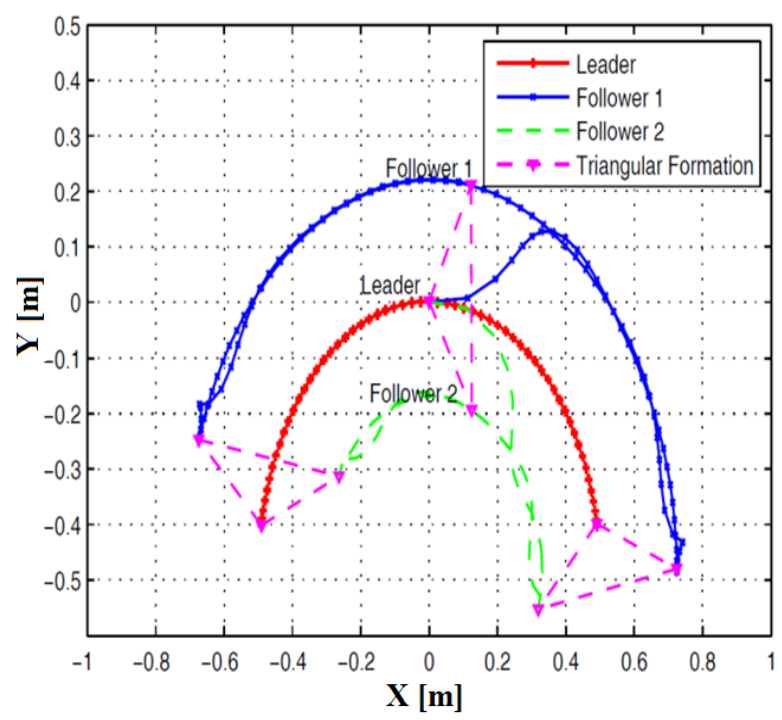

Figure 6: Semicircular trajectory of the leader robot with two follower robots which overall realize a triangular formation

The validation of the dynamic-static feedback technique, detailed in this paper, has been achieved using three Pololu mobile robots with a diameter $9.5 \mathrm{~cm}$. , a simple algorithm for tracking path for a trajectory desired in the leader robot has been implemented in the processing software aided by a simple web cam. This approach is inspired from the human vision of determining the deviation of the path by having knowledge of the path ahead by comparing the orientation of 
this one. This is a simple computational technique working on the pixel information of the image in comparison to other complex mathematical techniques available. The experimental results are showed in the Figure 7. One can see the trajectory for each robot and the triangular formation in Figure $7 \mathrm{a}$ (green line is for leader robot). The experimental scheme can be seen in Figure 7b.

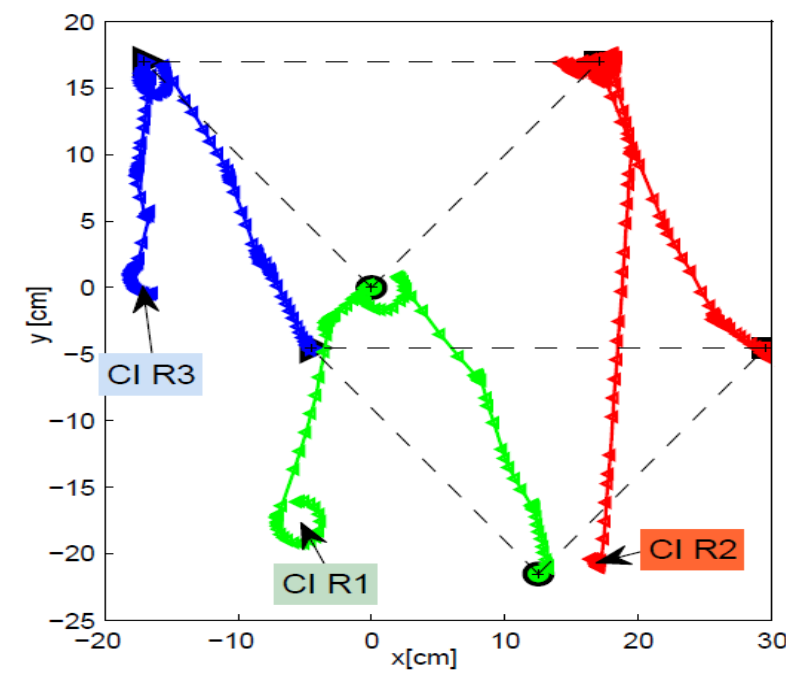

(a)

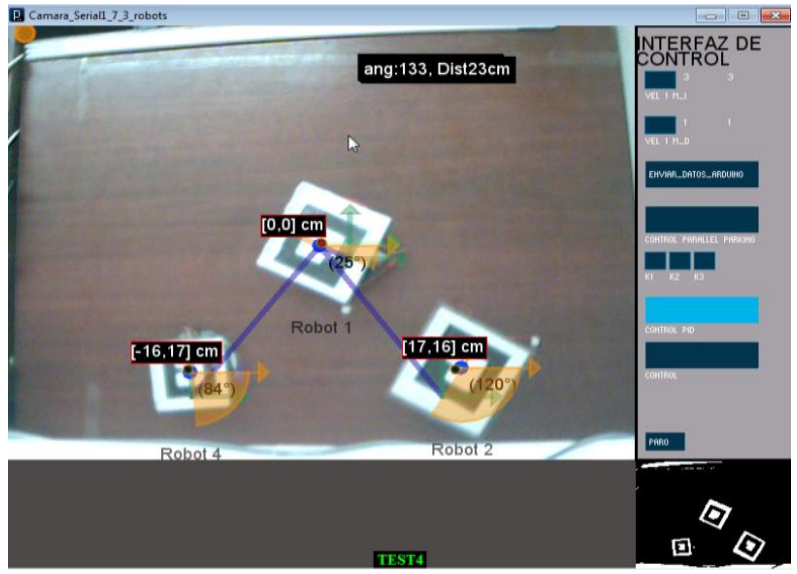

(b)

Figure 7: Experimental results. Robots paths, they are keeping the triangular formation (a). Experimental scheme shows the triangular formation too (b).

\section{CONCLUSIONS}

A comparative study of three different path tracking control laws for the formation of a group of nonholonomic mobile robots has been carried out satisfactorily. The dynamic-static feedback linearization control technique presents the best performance on the trajectory following on kinematics robots with circle path and three different paths maintaining a simple triangular formation of multiple robots. The simulations and experimental results showed that this technique presents also a stable tracking with smoother behaviour and avoiding discontinuities for tracking trajectory of the robot leader. This analysis can be extended to underactuated AUVs in future work.

\section{ACKNOWLEDGMENTS}

This project has been funded by the CONACyT-Mexico grant CB-169062 and also it has been partially funded by PROMEP: Redes Temáticas de Colaboración under the project titled: Fuentes de Energías Alternas and by the ECEST-SEP (Espacio Común de Educación Superior Tecnológica) program under the mobility scheme for professors and students, and Jesús Manuel Muñoz-Pacheco thanks to VIEP-BUAP-2013 for the financial support.

\section{REFERENCES}

[1] Gamage, G.W., Mann, G.K.I., Gosine, R.G., Formation Control of Multiple Nonholonomic Mobile Robots Via Dynamic Feedback Linearization, In Proceedings International Conference on Advanced Robotics, IEEEICAR 2009, pp. 1-6, 2009.

[2] Isidori A., Nonlinear control systems, $3^{\text {rd }}$ Edition, Springer-Verlag, 1995.

[3] C. Samson, "Time-varying feedback stabilization of carlike wheeled mobile robots," Int. J. of Robotics Research, vol. 12, no. 1, pp. 55-64, 1993.

[4] D. Rus, B. Donald, and J.Jennings, "Moving furniture with a team of autonomous robots," in Proc. IEEE/RSJ IEEE. Int. Conf. Intelligent Robots and Systems, pp. 556$561,1995$.

[5] M. Mataric, M. Nilsson, and K. Simsarian, "Cooperative multi-robot box pushing," in Proc. IEEE/RSJ IEEE. Int Conf. Intelligent Robots and Systems, pp. 235-242, 1995.

[6] T. Balch and R. Arkin, "Behaviour-based formation control for multirobot systems," IEEE Trans. on Robotics and Automation, vol. 14, no. 6, pp. 926-939, 1998.

[7] A. Das, R. Fierro, V. Kumar, J. Ostrowski, J. Spletzerm, and C. Taylor, "A vision-based formation control framework," IEEE Trans. on Robotics and Automation, vol. 18, no. 5, pp. 813-825, 2002.

[8] W. Ren, "Consensus based formation control strategies for multivehicle systems," In Proc. American Control Conference, June 2006.

[9] M.-Y. Chow, S. Chiaverini, and C. Kitts, "Guest Editorial introduction to the focused section on mechatronics in multirobot systems," IEEE/ASME Trans. Mechatronics, vol. 14, no. 2, pp. 133-140, 2009

[10] R. M. Bhatt, C. P. Tang, and V. N. Krovi, "Formation optimization for a fleet of wheeled mobile robots-A geometric approach," Robot. Auton. Syst., vol. 57, no. 1, pp. 102-120, 2009 .

[11] M. Egerstedt and X. Hu, "Formation constrained multiagent control," IEEE Trans. Robotics and Automation, vol. 17, pp. 947-951, 2001.

[12] J. P. Desai, J. Ostrowski and V. Kumar, "Controlling formations of multiple mobile robots," IEEE International Conference on Robotics \& Automation, Belgium, 1998.

[13] M. S. Carlos et al., "Coordinated control of mobile robots based on artificial vision," International Journal of Computers, Communication \& Control, vol. 1, no. 2, pp. 85-94, 2006.

[14] Tilbury D, R.M. Murray, S.S Satry, "Trajectory generation for the n-trailer problem using goursat normal form", IEEE Trans. on Automatic Control, vol. 40, no. 5, 1995. 